\title{
Learning Grammar in Confined Worlds
}

\author{
Graham Spinks, Ruben Cartuyvels, Marie-Francine Moens
}

\begin{abstract}
In this position paper we argue that modern machine learning approaches fail to adequately address how grammar and common sense should be learned. State of the art language models achieve impressive results in a range of specialized tasks but lack underlying world understanding. We advocate for experiments with the use of abstract, confined world environments where agents interact with the emphasis on learning world models. Agents are induced to learn the grammar needed to navigate the environment, hence their grammar will be grounded in this abstracted world. We believe that this grounded grammar will therefore facilitate a more realistic, interpretable and human-like form of common sense.
\end{abstract}

\section{Introduction}

It is generally well understood that humans create abstract models of the world from which they draw inferences [2,3]. A recent study in mice shows that visual inputs are encoded by predicting the impact of actions on the surroundings [7]. It makes sense that such world models are a byproduct of the necessity to deal with everyday tasks. The complex details of all possible interactions are abstracted to a level of detail that best suits the ability to navigate the world.

Most machine learning applications lack such interactions and reduce training to one or two objectives, e.g. achieve the best caption, achieve the most fluidity, etc. Some models excel at complex natural language benchmarks such as GLUE and SuperGLUE $[12,13]$ yet they fail to grasp basic, common sense. Studies have shown that these models exploit several fallible syntactic heuristics rather than reason about the underlying meaning [8]. A better goal would be to achieve a type of world understanding or common sense, which we describe as the ability to understand things as they really are. This implies that data is processed and understood with respect to a broader, physical context.

Graham Spinks $^{1} \cdot$ Ruben Cartuyvels ${ }^{1} \cdot$ Marie-Francine Moens $^{2}$

KU Leuven, e-mail: ${ }^{1}$ : first.last@cs.kuleuven.be, ${ }^{2}$ : sien.moens@cs.kuleuven.be 
We posit that 'making oneself understood' is a task that humans learn as part of the objectives in everyday life. We learn our language from interacting with teachers (humans, books, or even television) and with the world, because it inherently lets us navigate the world better and achieve our goals as humans.

Whereas animals possess a form of common sense, human world knowledge and understanding far exceeds that of animals. While common knowledge is often implicit in communication, and most likely results from world interaction, humans do use language to transmit complex information that relates to the world. It is therefore sensible to study how grammar and common sense relate to each other. It is interesting to see that language has developed across the world in many different ways, but that there are common factors. These universals include basic notions such as 'verbs' or complex ideas as 'Universal Grammar' [1]. The latter states that all humans are born with the capacity to learn and understand grammar.

Taken together, the ideas in this paragraph imply that human grammar is grounded in world interaction too, and we postulate that the resulting structured language facilitates the navigation of world models and common sense. Grammar in our view provides a set of tools that can be used to relate concepts and objects in our internal world models. This increases the capacity of internal models to express the necessary complexity to navigate the world. These models could therefore facilitate a more elaborate common sense understanding. To extrapolate this idea to machine learning, one could deploy models that are capable of learning grammar in an environment that induces them to learn it.

\section{From Complexity To Controlled Experiments}

Current approaches to NLP tend to rely on more and more complex datasets that are solved with larger and larger models. As noted by [6], meaning representations are mostly constructed following the distributional hypothesis [4], grounding symbols in other symbols instead of in reality. As a consequence, models learn to exploit word co-occurrences and unintended features in the dataset. This leads to nontransparent calculations and outputs that conflict with human understanding of the world.

An interesting avenue is to place models in virtual or physical environments that are affected by a temporal component. This idea has been expressed by other researchers, yet the push towards higher complexity has perhaps complicated this line of investigation. Many controlled experiments are possible which could advance our understanding of language learning. By setting up a 'confined world' that follows the virtual embodiment principles proposed by [6], different priors and models can be investigated. Rather than setting up 3D physical or photo-realistic simulation environments with complex interactions as in [5, 11], we suggest abstracting the physical world to a set of parameters of increasing complexity.

Some work in this direction has been done, where e.g. the emergence of language was studied in a cooperative multi-agent game in an abstracted environment [9]. Many more abstractions, tasks, situations, agents and interactions (e.g. humancomputer vs. computer-computer) remain to be explored. [10] proposes agent-based 
interactions to test and sniff out the cognitive mechanisms that lead to the development of languages. Whereas this approach advocates for a semantic representation formalism based on second-order intentional logic, we believe that such agents should form their own implicit models of interactions and meaning. Our suggestion is therefore to focus on architectures that mirror the innate capability of brains to learn syntactical and morphological complexities of language [1] rather than relying on pre-specified symbolic formalisms. Artificial agents could, for example, exploit model architectures that explicitly detect (dis)agreement between language morphology and the state of the world. As a concrete example, for the sentence "The apples are falling", a model might be designed to detect that the sentence contains a plurality of apples as expressed by the subject and verb, and align it with observations of multiple falling apples from the evolution of the world state.

Concretely we propose abstracted environments that contain the following: spatial and temporal axes (coordinates, time), perception (vision and/or touch), grammar (a set of simplified rules to convey messages based on language universals), interaction and objectives. Objectives should prompt agents to implicitly learn a basic model of the world and the language rules to navigate it successfully. In these confined worlds, the importance of teachers, including whether their language supervision is needed at all, as well as the elements needed to express language in relation to the world can be investigated. Methods could focus on different model structures and learning algorithms that are better suited to induce grammar given different types of interactions. Comparing various types of objectives could lead to a better understanding of the implicit goals that align with language learning. We believe such experimentation will lead to a better understanding and new research directions, and enable valuable applications.

Acknowledgements This work is part of the CALCULUS project at the KU Leuven, funded with the European Research Council Advanced Grant H2020-ERC-2017-ADG 788506.

\section{References}

[1] Chomsky N (2000) New horizons in the study of language and mind. Cambridge University Press

[2] Forrester JW (1971) Counterintuitive behavior of social systems. Technological Forecasting and Social Change 3:1-22

[3] Ha D, Schmidhuber J (2018) Recurrent world models facilitate policy evolution. In: Advances in Neural Information Processing Systems 31, pp 24502462

[4] Harris ZS (1954) Distributional structure. Word 10(2-3):146-162

[5] Hermann KM, Hill F, Green S, Wang F, Faulkner R, Soyer H, Szepesvari D, Czarnecki WM, Jaderberg M, Teplyashin D, et al (2017) Grounded language learning in a simulated 3d world. arXiv preprint arXiv:170606551 
[6] Kiela D, Bulat L, Vero AL, Clark S (2016) Virtual embodiment: A scalable long-term strategy for artificial intelligence research. arXiv preprint arXiv:161007432

[7] Leinweber M, Ward DR, Sobczak JM, Attinger A, Keller G (2017) A sensorimotor circuit in mouse cortex for visual flow predictions. Neuron 95(6):1420 1432

[8] McCoy RT, Pavlick E, Linzen T (2019) Right for the wrong reasons: Diagnosing syntactic heuristics in natural language inference. arXiv preprint arXiv:190201007

[9] Mordatch I, Abbeel P (2018) Emergence of grounded compositional language in multi-agent populations. In: Thirty-Second AAAI Conference on Artificial Intelligence

[10] Steels L (2016) Agent-based models for the emergence and evolution of grammar. Philosophical Transactions of the Royal Society B: Biological Sciences 371(1701):20150,447

[11] de Vries H, Shuster K, Batra D, Parikh D, Weston J, Kiela D (2018) Talk the walk: Navigating new york city through grounded dialogue. arXiv preprint arXiv:180703367

[12] Wang A, Singh A, Michael J, Hill F, Levy O, Bowman SR (2018) Glue: A multi-task benchmark and analysis platform for natural language understanding. EMNLP 2018 pp 353-355

[13] Wang A, Pruksachatkun Y, Nangia N, Singh A, Michael J, Hill F, Levy O, Bowman SR (2019) Superglue: A stickier benchmark for general-purpose language understanding systems. Advances in Neural Information Processing Systems 32 pp 3261-3275 